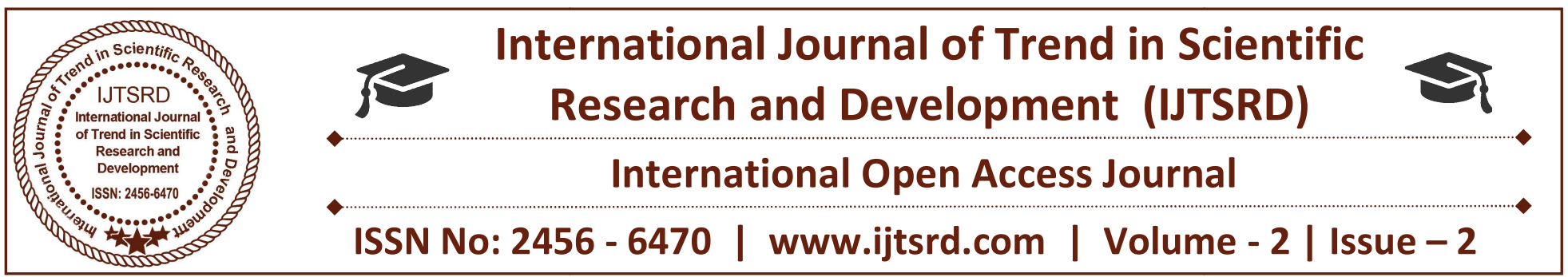

\title{
4G Wireless Technology
}

\author{
S. Atchaya, S. Selvanayaki, S. Deepika \\ Computer Science Department, Sri Krishna Adithya College of Arts and Science \\ Coimbatore, Tamil Nadu, India
}

\begin{abstract}
Mobile Communication has been developed rapidly since last few decades. The growth of the wireless broadband technologies in the modern years was the answer of increasing demand for mobile Internet and wireless multimedia application such as live TV, live Movies, video conferencing etc. Mobile communication plays a vital role in telecommunication industry. During a common wide area radio access technology and supple network architecture WiMAX and LTE has facilitate convergence of mobile and fixed broadband network
\end{abstract}

Keywords: WiMAX, LTE (Long Time Evolution), OFDMA (Orthogonal Frequency Division Multiplex Access), MIMO (Multiple Input Multiple Output), MME (Mobility Management Entity)

\section{INTRODUCTION}

The mobile and wireless communication technologies have been enhancing rapidly day by day. Gadgets continue to shrivel in size and at the same time rising in processing power. Users generally insist in more sophisticated and worthwhile applications. Hence, capacity improvement is the paramount necessity in wireless communications. The evolution of various mobile services initiating from the $1 \mathrm{G}$ to $4 \mathrm{G}$ is begun as follows:

1G: First generation (1G) was a wireless network which basically constituted an analog cellular system along with circuit switched network architecture. These wireless networks only supported basic voice telephony and were mainly confronted by low capacity and limited coverage region. Hence in telecommunications sector an increased requirement for high frequency ranges paved the way for the development of digital transmission techniques from analog transmission techniques

2G: In the early $1990 \mathrm{~s}$, second generation (2G) wireless technology arrived to meet the capacity requirements of burgeoning voice plus telephony, limited circuit switched data services and text messaging. If This technology utilized digital transmission system which is capable of compressing the signal much more efficiently and effectively as compared to analog system and at the same time allows the transmission of more packets in the same bandwidth with lesser power

2.5G: $2.5 \mathrm{G}$, an interim step being taken after $2 \mathrm{G}$ and prior to $3 \mathrm{G}$, was basically an improvement of the two chief $2 \mathrm{G}$ technologies. This technology provided an enhanced capacity on the $2 \mathrm{G}$ RF (radio frequency) channels and also presented higher throughput data rates, up to $384 \mathrm{kbps}$.

3G: $3 \mathrm{G}$, third generation of mobile and wireless technology, supersedes $2 \mathrm{G}$ technology and precedes $4 \mathrm{G}$ technology. $2.5 \mathrm{G}$ was a transitory bridge between $2 \mathrm{G}$ and $3 \mathrm{G}$ for providing high data rates. Hence $3 \mathrm{G}$ wireless technology was introduced for bestowing higher data-transmission speeds, superior network capacity and more sophisticated and enhanced network services. In May 2001 NTT DoCoMo launched the first pre-commercial $3 \mathrm{G}$ network, branded as FOMA, in Japan. Subsequently after the first pre-commercial launch, NTT DoCoMo launched 
the first commercial $3 \mathrm{G}$ network in Japan in Oct. 2001.

4G: 4G, the fourth-generation of wireless service, is an enhancement from $3 \mathrm{G}$ and is presently the most extensive, widespread, expeditious and high-speed wireless service. Presently $4 \mathrm{G}$ is available only in limited regions. $4 \mathrm{G}$ wireless service has been devised to deliver high speed irrespective of the technology which drives 4G. For instance Sprint employs a technology called WiMax for its $4 \mathrm{G}$ services, whereas Verizon Wireless employs Long Term Evolution, or LTE. On an average, 4G wireless technology is expected to provide data rates from four to ten times higher than today's conventional $3 \mathrm{G}$ networks.

\section{FOURTH GENERATION NETWORKS}

The $4 \mathrm{G}$ is the most innovative wireless technology which has replaced the $3 G$ systems. The vital characteristics of the $4 \mathrm{G}$ networks include accessing information with a flawless connection anytime, anywhere.

Enhanced Features of 4G Wireless Technology äre as follows;

$>$ Larger bandwidth - higher data rates.

$>$ Terminal Heterogeneity and Heterogeneity.

$>$ Smoother and quicker handoff.

$>$ WLAN for hot spots, an extension of $2 \mathrm{G}$ and $3 \mathrm{G}$.

$>$ Better scheduling and call admission control techniques.

$>$ Global roaming and inter-working among various other access technologies.

$>$ Supports interactive multimedia, video, wireless internet, voice and various other

\section{BENEFITS AND CHALLENGES}

\section{A. Benefits of $4 \mathrm{G}$ networks}

The benefits of $4 \mathrm{G}$ networks assist in ensuring a larger range of services and use-cases. However, the commercial models and eco-systems have not yet been established that are required in driving adoption from a user and service provider perspectives.

Technology Performance Improvement:

Delivers higher uplink and downlink throughput besides lesser latency and network capabilities. It has been universally believed that there will be a prolong growth in mobile data traffic significantly in the coming years. It is also a matter of fact that the majority of the core transport and throughput bottlenecks will undoubtedly be delivered by the technology itself despite of the 4G technology used in comparison to $3 \mathrm{G}$. $4 \mathrm{G}$ technologies provide at least two times more effective and efficient use of spectrum, enhanced support for real-time applications, and greater max speeds. Though there exist further network and capacity confronts such as edge or gateway management, signalling management which are needed to be fully addressed to increase benefits from the upgrade

Mobile Application Enablement:

New It enables new mobile applications to enhance the existing ones (Streaming Music). Several 4G services such as digital storage or smart home monitoring will get enhanced by the improved $4 \mathrm{G}$ bandwidth and latency. Other services such as MMS, digital picture frames and various near-field communication applications will notice no significant improvement in riding on a $4 \mathrm{G}$ network. Hence, it is very crucial to have a very close look at the services and applications which are likely to be enhanced by 4G advancements. We can see that services which gain the most from the 4G technology's deployment are video streaming, MMOG/gaming and expertise applications such as interactive learning

Addressable Device Expansion:

Network potentials and chipset scale could expand the connectivity to various innovative gadgets. Handset technologies persist in evolving along a huge range of features and value added services by means of smart phones and more specialized gadgets. A carrier controlled service experience has been conventionally supported by the Terminal operating model. Commercial operating systems such as Windows Mobile or RIM have attracted heavy data users and hence fostered network congestion by reducing some control In addition, the increasingly growing open eco-systems, further enabled by $4 \mathrm{G}$, offer a challenging opportunity for operators since third parties develop services, applications and customization tools in order to meet user needs. Gadgets are becoming highly configurable because of open standards and more expertise gadgets such as net books, eReaders, tablets etc. are coming into the market. To meet lesser user segment needs we believe that vendors must think of a micro-segmentation based device roadmap; various new distribution 
channels are requisite to support the acceptation of Converged Mobile Gadgets and 4G applications

Differentiated Customer Experience: It enables in managing the user expectation and experience with new features and services. We consider the user's experience in gaining a profound understanding of how these services are completely facilitated and how it mingles into the fabric of our living, the necessity or capability to deploy expertise or configured gadgets to support enhancement, and finally, how to make money and when to share the income from the service delivery. Till now, it has been inadequate in understanding the experience of a $4 \mathrm{G}$ user and it is uncertain that how greatly the user experience will alter as many more and various $4 \mathrm{G}$ services arrive.

\section{QUALITY OF SERVICE}

Traffic generated by the different services will not only increase traffic loads on the networks, but will also require different quality of service (QoS) requirements.

Providing QoS guarantees in $4 \mathrm{G}$ networks is a nontrivial issue where both QoS signaling across different networks and service differentiation between mobile flows will have to be addressed.

One of the most difficult problems that are to be solved, when it comes to IP mobility, is how to insure the constant QoS level during the handover. Depending on whether the new access router is in the same or some other subnetwork, we recognize the horizontal and vertical handover.

However, the mobile terminal cannot receive IP packets while the process of handover is finished. This time is called the handover latency.

Handover latency has a great influence on the flow of multimedia applications in realtime. Mobile IPv6 have been proposed to reduce the handover latency and the number of lost packets.

The field "Traffic Class" and "Flow Label" in IPv6 eader enables the routers to secure the special QoS for specific packet series with marked priority.

\section{SECURITY}

The heterogeneity of wireless networks complicates the security issue. Dynamic reconfigurable, adaptive, and lightweight security mechanisms should be developed.
Security in wireless networks mainly involves authentication, confidentiality, integrity, and authorization for the access of network connectivity and QoS resources for the mobile nodes flow.

\section{AAA (Authentication Authorization Auditing)} protocols provide a framework for such suffered especially for control plane functions and installing security policies in the mobile node such as encryption, decryption and filtering.

Users will benefit from faster access to a range ofbroadband multi-media services reasonable QoS and lower cost.

\section{CONCLUSION}

As the history of mobile communications shows, attempts have been made to reduce a number of technologies to a single global standard.

Projected $4 \mathrm{G}$ systems offer this promiseof a standard that can be embraced worldwide through its key concept of integration.

Futurewireless networks will need to support diverse IP multimedia applications to allow sharing ofresources among multiple users.

There must be a low complexity of implementation and an efficient means of negotiation between the end users and the wireless infrastructure. Fourth generation promises to fulfill the goal of PCC (personal computing and communication) a vision that affordably provides high data rates everywhere over awireless network.

4G is expected to be launched by 2010 and the world is looking forward

for the most intelligent technology that would connect the entire globe.

\section{Reference}

1) Bill Krenik “4G Wireless Technology:When will it happen? What does it offer?" IEEE

2) R. Mayuri, P. Manish " 4G Wireless Technology: A Survey Paper". In: Proceedings of the National Conference "NCNTE-2012" at Fr. C.R.I.T., Vashi, Navi Mumbai

3) R. Mayuri, P. Manish “ 4G Wireless Technology: A Survey Paper". In: Proceedings of the National Conference "NCNTE-2012" at Fr. C.R.I.T., Vashi, Navi Mumbai 